\title{
A prospective microbiological surveillance in a teaching Italian Hospital; microbial isolations and in vitro antimicrobial susceptibility levels, and their modifications over time
}

\author{
Roberto Manfredi
}

From $16^{\text {th }}$ International Symposium on HIV and Emerging Infectious Diseases

Marseille, France. 24-26 March 2010

\section{Background}

A prospective microbiological surveillance of microorganisms isolated at a reference hospital and their antimicrobial susceptibility, is of paramount importance in the awareness of evolving local epidemiology.

\section{Methods}

The trend of microbial isolations from patients (p) hospitalized in the last year 2008 (January 1, 2008-December 31,2008 ), is reported on quarterly basis, together with the related antimicrobial sensitivity testing. Surveillance cultures and multiple isolations of the same organism(s) from one p within one month of hospitalization, were excluded.

\section{Results}

4,906 overall evaluable pathogens were isolated in the last 12 mo, with E. coli (1,279), E. faecalis (596), P. aeruginosa (430), and S. aureus (365), as prevailing organisms. Among blood cultures (1,421 isolates), a major role was played by Staphylococci as a whole (688), followed by Enterobacteriaceae (253), and Enterococci (77). With regard to the overall susceptibility rates, methicillin-resistant $S$. aureus accounted for a mean $42 \%$, while methicillin resistance was greater for $S$. epidermidis (>70\%), with a slight reduction compared with the year 2007 figures. Substantially ameliorated sensitivity levels were found among Enterococci, with only 7 "VRE" strains recorded (versus 17-21 strains per year, during 2005-2007); a slight increase of resistance towards glycopeptides was found, reaching $3.1 \%$ of isolates in the last

Correspondence: Roberto.manfredi@unibo.it

Infectious Diseases, University of Bologna, Bologna, Italy
3 mo. A $25-38 \%$ resistance rate to macrolides was found among streptococci, without appreciable temporal variations. Among Gram-negative organisms, extended spectrum beta-lactamase production regarded $31.8 \%$ of overall E. coli isolates, and $39.6 \%$ of other Enterobacteriaceae, with a slight increase compared with the year 2007. P. aeruginosa showed an stable resistance pattern to penicillins-cephalosporins (including those protected by beta-lactamase inhibitors), carbapanems, fluoroquinolones, and aminoglycosides (ranging from $55 \%$ to $75 \%$ of strains), but remained full susceptible to colistin. Stenotrophomonas maltophilia confirmed its extensive resistance spectrum, but remained $90-100 \%$ sensitive to cotrimoxazole and colistin, as well as Acinetobacter spp., which showed a favorable susceptibility rate $(60-100 \%)$ to aminoglycosides, piperacillin-tazobactam, and colistin.

\section{Discussion}

An active bacteriological surveillance may notably add to the knowledge of local epidemiological figures and antimicrobial sensitivity trends, and plays a major role when planning surveillance measures, chemoprophylaxis, and empiric antimicrobial treatment, on both local and regional basis.

Published: 11 May 2010

doi:10.1186/1742-4690-7-S1-P172

Cite this article as: Manfredi: A prospective microbiological surveillance in a teaching Italian Hospital; microbial isolations and in vitro antimicrobial susceptibility levels, and their modifications over time. Retrovirology 2010 7(Suppl 1):P172. 